\title{
ATIVIDADE FÍSICA COMO ADJUNTO TERAPÊUTICO PARA PACIENTES PSIQUIÁTRICOS COM ADOECIMENTO MENTAL SEVERO: REVISÃO DA LITERATURA
}

\author{
PHYSICAL ACTIVITY AS A THERAPEUTIC ADJUNCT TO PSYCHIATRIC PATIENTS WITH SEVERE \\ MENTAL ILLNESS: A LITERATURE REVIEW \\ ACTIVIDAD FÍSICA COMO COADYUVANTE TERAPÉUTICO PARA PACIENTES PSIQUIÁTRICOS \\ CON ENFERMEDAD MENTAL SEVERA: REVISIÓN DE LA LITERATURA
}

\author{
Maria Cristina Zago* \\ Bruneide Menegazzo Padilha*
}

\begin{abstract}
RESUMO
Existe a carência de esclarecimentos mais precisos sobre as repercussões psíquicas da prática de atividade física $(\mathrm{AF})$ nos serviços de saúde mental para pessoas com adoecimento mental severo (severe mental illness; SMI). $\mathrm{O}$ objetivo desta revisão foi investigar a $\mathrm{AF}$ como adjunto terapêutico para pacientes com SMI. Foram usadas as bases de dados EBSCOHost, PsycInfo, SportDiscus, Medline, PsyArticles. Estudos qualitativos e quantitativos publicados entre 2000 e 2014 foram inclusos e também literatura anterior considerada relevante. As investigações relatam que a AF pode contribuir para a melhora de humor, estado de alerta, concentração, padróes de sono e sintomas psicóticos; aliviar sintomas secundários como baixa autoestima e retraimento social. Há evidência também de benefícios psicológicos da $\mathrm{AF}$ em pacientes depressivos. Os pacientes veem a AF positivamente e gostariam de ser ativos e melhorar a saúde mental. Barreiras identificadas pelos pacientes foram: impacto do adoecimento e efeitos de medicação, fadiga, ganho de peso por medicação, medo de discriminação e questôes relativas à segurança e falta de suporte profissional. O contexto grupal aparece como um ambiente favorecedor da prática de AF. A literatura considera a $\mathrm{AF}$ como uma intervenção que pode ser útil para a promoção da saúde mental e bem-estar, portanto uma estratégia em reabilitação psicossocial para pessoas com SMI. As conclusóes dos estudos se restringem a um âmbito descritivo, são limitadas, carecem de uma metodologia de pesquisa precisa em alguns casos, revelando a necessidade de novos estudos.
\end{abstract}

Palavras-chave: Saúde mental. Atividade física. Grupo.

\footnotetext{
Texto recebido em 12 de março de 2014 e aprovado para publicação em 14 de maio de 2015.

Doutora e mestra em Psicologia pela Pontifícia Universidade Católica de Campinas, psicóloga, grupanalista, presidente da ONG Saúde Mental do Brasil (SAMBRA), docente das Faculdades Atibaia (FAAT). E-mail: mariazago916@gmail.com.

** Doutora e mestra em Psicologia pela Pontifícia Universidade Católica de Campinas, supervisora da rede de Saúde Pública do Município de Campinas-SP, docente do Centro de Formação e Assistência à Saúde (Cefas), membro da diretoria da Sambra. E-mail: bruneidepadilha@uol.com.br.
} 


\begin{abstract}
There is a lack of more precise clarification of the psychological impact of physical activity (PA) in Mental Health Services for people with severe mental illness (SMI). This review aims to investigate physical activity as a therapeutic adjunct for patients with SMI. Data sources: EBSCOHost, PsycInfo, SPORTDiscus, Medline, PsyArticles. Quantitative and qualitative articles published between 2000-2014 were sourced. The studies report that PA can contribute to improve mood, alertness, concentration, sleep patterns and psychotic symptoms. It can relieve secondary symptoms such as low self-esteem and social withdrawal. There is also evidence of psychological benefits of PA in depressed patients. Patients see PA positively and would like to be active and improve mental health. Barriers to uptake of PA were identified: impact of the illness and effects of medication, fatigue, weight gain by medication, fear of discrimination and safety issues and lack of professional support. The group context appears as an environment that promotes the practice of PA. The literature considers AP as an intervention that can be useful for promoting mental health, well-being, therefore a psychosocial rehabilitation strategy for people with SMI. The conclusions of the studies are restricted to a descriptive level, are limited, and lack an accurate methodology of research in some cases revealing the need for future studies.
\end{abstract}

Keywords: Mental health. Physical activity. Group.

\title{
RESUMEN
}

Existe la carencia de aclaraciones más precisas sobre las repercusiones psíquicas de la práctica de actividad física (AF) en los Servicios de Salud Mental para personas con enfermedad mental severa (severe mental illnes; SMI). El objetivo de esta revisión fue investigar la AF como coadyuvante terapéutico para pacientes con SMI. Fueron usadas las bases de datos EBSCOHost, PsycInfo, SportDiscus, Medline, PsyArticles. Se incluyeron estudios cualitativos y cuantitativos publicados en el período entre 20002014 y la literatura anterior considerada relevante. Los estudios relatan que la AF puede contribuir a la mejora del estado de ánimo, estado de alerta, concentración, patrones de sueño y síntomas psicóticos; aliviar los síntomas secundarios como baja autoestima y retraimiento social. También hay evidencia de beneficios psicológicos de la AF en pacientes depresivos. Los pacientes ven la AF de forma positiva y desean ser activos y mejorar la salud mental. Las barreras identificadas por los pacientes fueron: impacto de la enfermedad y efectos de la medicación, fatiga, aumento de peso por medicación, miedo a la discriminación y cuestiones relativas a la seguridad y falta de soporte profesional. El contexto grupal aparece como un entorno favorecedor de la práctica de AF. La literatura considera la AF como una intervención que puede ser útil para la promoción de la salud mental y el bienestar, por lo tanto una estrategia en rehabilitación psicosocial para las 
personas con SMI. Las conclusiones de los estudios se restringen a un ámbito descriptivo, son limitadas, carecen de una metodología de investigación precisa en algunos casos revelando la necesidad de nuevos estudios.

Palabras clave: Salud mental. Actividad física. Grupo.

\section{INTRODUÇÃO}

0 $s$ benefícios da prática de atividade física $(\mathrm{AF})$ vêm sendo largamente discutidos na mídia e na literatura em diversas áreas do conhecimento (World Health Organization, 2010). Contudo se nota um interesse crescente dos pesquisadores das repercussóes da prática de $\mathrm{AF}$ em pacientes psiquiátricos na década de 1990 (Faulkner \& Biddle, 1999; Faulkner \& Sparkes, 1999; Hutchinson, Skrinar, \& Cross, 1999; Skrinar, Unger, Hutchinson, \& Faigenbaum, 1992) e no início do século XXI (Adamoli \& Azevedo, 2009; Carter-Morris \& Faulkner, 2003; Faulkner, Cohn, \& Remington, 2006; Giuliani, Micacchi, \& Valenti, 2005; McDevitt, Robinson, \& Forest, 2005; Meyer \& Broocks, 2000; Richardison, et al., 2005; Trivedi, Greer, Grannemann, Chambliss, \& Jordan, 2006; Ussher, Stanbury, Cheeseman, \& Faulkner, 2007).

Alguns estudos discutem os benefícios da prática de exercícios regulares para indivíduos submetidos a tratamento psiquiátrico e abordam o impacto da prática de AF do ponto de vista organicista, no sentido da prevenção de patologias advindas dos efeitos colaterais do tratamento medicamentoso e do estilo de vida dessa população, por vezes sedentário (Faulkner et al., 2006; Leibovich \& Iancu, 2007; McDevitt et al., 2005; Scott \& Happell, 2011; Smith et al., 2007). No entanto se observa a carência de esclarecimentos mais precisos sobre as consequências da prática de $\mathrm{AF}$ nos serviços de saúde mental para pessoas com doença mental severa severe mental illness, (SMI), especialmente no que se refere às repercussões psíquicas do ponto de vista dinâmico.

Adultos com um adoecimento mental severo são pessoas: (1) idade de 18 anos ou mais, (2) que atualmente, ou em qualquer momento durante o ano passado, (3) teve um distúrbio mental, comportamental ou emocional diagnosticável de duração suficiente para cumprir os critérios de diagnóstico especificadas no Manual Diagnóstico e Estatístico de Transtornos Mentais (DSM)-III-R, (4) que resultou em prejuízo funcional que interfere substancialmente com os limites de uma ou mais atividades principais da vida [...] Todos esses transtornos têm características episódicas, recorrentes ou persistentes, no entanto eles variam em termos de gravidade e efeitos incapacitantes (Federal Register, 1993, apud Insel, 2013 tradução nossa). ${ }^{1}$

\footnotetext{
"'Adults with a serious mental illness are persons: (1) age 18 and over, (2) who currently or at any time during the past year, (3) have a diagnosable mental, behavioral, or emotional disorder of sufficient duration to meet diagnostic criteria specified within the Diagnostic and Statistical Manual of Mental Disorders (DSM)-III-R, (4) that has resulted in functional impairment which substantially interferes with or limits one or more major life activities...All of these disorders have episodic, recurrent, or persistent features; however, they vary in terms of severity and disabling effects.' Federal Register Volume 58 No. 96 published Thursday May 20, 1993, pages 29422-29425.”
} 
Nesse sentido, este estudo investigou a prática de atividade física em saúde mental como adjunto terapêutico para pacientes psiquiátricos com doença mental severa.

\section{DESENHO METODOLÓGICO}

Neste estudo, AF é considerada como caminhada e exercício estruturado, incluindo atividade física em grupo ou esporte (Soundy, Faulkner, \& Taylor, 2007). A pesquisa dos artigos foi conduzida por meio da base de dados EBSCOHost, ${ }^{2}$ PsycInfo, SPORTDiscus, Medline, PsyArticles: termos de indexação: mental health (saúde mental), severe mental illness (doença mental severa), physical activity (atividade física), exercise (exercício), group psychoterapy (psicoterapia de grupo), group analysis (grupanálise), psychoanalysis (psicanálise). Em relação à categoria mental health (saúde mental), usou-se a qualificação severe (severa) como predecessor. Ao mesmo tempo, os termos schizophrenia (esquizofrenia) e psychosis (psicose) foram usados na busca. O termo depression (depressão) isolado representa artigos relativos à depressão leve ou moderada. Assim, apenas os trabalhos referentes à severe (severa) ou depressão bipolar foram incluídos. Os artigos relativos à doença de Alzheimer e demência foram excluídos, pois não se enquadravam na proposta desta pesquisa. Dessa forma, foram inclusos artigos publicados em periódicos científicos no período entre 2000 e 2014 e literatura anterior considerada relevante (texto completo). As intervenções pertinentes a exercício ou atividade física (isoladas ou como parte de um programa que envolvia outras intervenções) foram selecionadas, o que está de acordo com a definição de atividade física que norteia este estudo (Soundy, Faulkner, \& Taylor, 2007). Primeiramente, dois investigadores avaliavam os títulos e resumos das publicações encontradas para verificar se atendiam ou não aos critérios de inclusão. Apenas quando houve a concordância entre os dois investigadores sobre a adequação do artigo científico ao objetivo deste estudo é que as informações pertinentes foram acessadas e anexadas. Procurou-se também fazer uma busca manual por estudos potencialmente pertinentes localizados nas listas de referência dos artigos selecionados, notadamente os de revisão sistemática da literatura, os quais talvez não tivessem sido encontrados na pesquisa às bases de dados. Em seguida, partiu-se para a procura direcionada dos referidos artigos considerados relevantes a este estudo. No total, 41 artigos foram selecionados para compor esta revisão, segundo os critérios de inclusão e exclusão descritos anteriormente.

\footnotetext{
Empresa irmã da EBSCO Information Services, a EBSCO Publishing é o fornecedor do EBSCOhost ${ }^{\circledR}$, o recurso eletrônico pago mais utilizado por bibliotecas ao redor do mundo. A EBSCO Publishing é o principal agregador de bases de dados do mundo, oferecendo uma suíte de mais de 200 bases de dados de pesquisa em texto completo e resumo. Recuperado a partir de: http://www2.ebsco.com/pt br/ProductsServices/ehostdatabases/Pages/index.aspx.
} 
Os artigos encontrados neste período têm uma vinculaçãoàs áreas de Psiquiatria, Saúde Mental, Medicina Esportiva, Enfermagem, Psicologia, Psicologia do Esporte e Ciência do Esporte. Nota-se que os trabalhos aludidos tendem a usar o termo SMI; adoecimento mental severo), ou seja, uma linguagem própria da nosografia psiquiátrica. ${ }^{3}$ Entende-se que a escolha desse termo SMI facilita o diálogo internacional entre as diferentes áreas do conhecimento científico que transitam no âmbito da saúde mental. Embora haja o reconhecimento dos benefícios do exercício físico (Adamoli \& Azevedo, 2009; Broocks \& Sommer, 2005; Broocks, 2005; Carter-Morris, \& Faulkner, 2003; Faulkner \& Carless, 2006; Faulkner et al., 2006; Giuliani, Micacchi, \& Valenti, 2005; Leibovich \& Iancu, 2007; McDevitt et al., 2005; Meyer \& Broocks, 2000; Richardison, et al., 2005; Trivedi et al., 2006; Ussher et al., 2007) as conclusóes dos estudos se restringem a um âmbito descritivo, são limitadas em termos metodológicos. Existe uma escassez de pesquisas em relação a esse tema, como aponta a literatura. Especificamente, estudos que norteiem a estruturação de programas específicos de $\mathrm{AF}$ para essa população, a fim de que esta prática figure como tratamento coadjuvante efetivo para indivíduos com SMI (Giuliani, et al., 2005; Richardison et al., 2005; Ussher et al., 2007). Uma das explicações apontadas para a carência de publicaçôes refere-se à dificuldade em conduzir pesquisa sobre AF no contexto de SMI em termos de acesso a subjetividade desses pacientes. Ao mesmo tempo, comorbidades e o uso de medicação podem afetar a resposta à AF o que torna problemático o delineamento das conclusões (Carless \& Douglas, 2008). Por outro lado, a heterogeneidade das intervenções e, por vezes, amostras pequenas utilizadas nos estudos limitam conclusões e evidenciam a necessidade de pesquisas futuras (Vancamfort, et al., 2012).

\section{DISCUSSÃO}

\subsection{Prática de AF em programas individuais e em situação de grupo}

Existem indagações, por exemplo, a respeito da aderência dos pacientes a programas individuais de AF em relação a outros que são desenvolvidos em grupo. Pontua-se que, embora o grupo se configure num espaço de baixo custo para o desenvolvimento dessas atividades, a disponibilidade do coordenador em dar atenção individualizada estaria dificultada, o que, segundo Giuliani et al. (2005) e Richardison et al. (2005), desempenha papel importante nesse processo interventivo. Estudando-se o impacto de um programa estruturado de exercícios no bem-estar físico e psíquico de pacientes com esquizofrenia ou demais psicoses, considerou-se relevante a abordagem grupal na intervenção

3 Termo usado frequentemente na clínica psiquiátrica e em muitos documentos de políticas governamentais em todo mundo (Smith et al., 2007). 
desenvolvida em termos de incremento de motivação e prazer em tomar parte (Fogarty \& Happell, 2005). Os benefícios da prática de AF na situação de grupo não foram devidamente explorados pela literatura. Embora esse artigo não seja recente, apresenta um exemplo de uma proposta de tratamento com orientação psicodinâmica para pacientes psiquiátricos, Evening Treatment Program, que continha a prática de exercício em dois grupos:

a) uma versão modificada do voleibol; e

b) exercícios aeróbicos (incluía também a dança).

Os autores apontam, como benefícios potenciais, a redução da tensão e o relaxamento; favorecimento da descoberta de que os pacientes podem participar e experienciar a prática de AF como prazerosa e desenvolver habilidades; a exploração dos limites corporais e internalização de uma imagem corporal saudável; favorecer processos de identificação com os terapeutas; experienciar fazer parte de um "time" e propiciar um ambiente confiável para a emergência e elaboração de conflitos (O'Kelly, Piper, Kerber, \& Fowler, 1998). Zago \& Terzis (2012) reportaram a importância da prática de AF em grupo segundo a técnica grupal chamada, Grupo de Atividades Físicas, no tratamento de pacientes com SMI em uma unidade de internação (inpatients: pacientes que permanecem no hospital enquanto recebem tratamento) de um serviço público de saúde mental do Estado de São Paulo, Brasil. Investigou-se a prática de AF como fator atenuante dos sintomas relativos ao adoecimento mental severo em situação de tratamento intensivo, segundo abordagem psicanalítica e grupanalítica (psicoterapia de grupo de base analítica). O grupo era aberto, heterogêneo quanto ao diagnóstico, gênero e idade. A técnica grupal, Grupo de Atividades Físicas, compreendia três momentos principais:

a) caminhada (de ida e volta do serviço ao centro de convivência);

b) atividade física coletiva; e

c) momento simbólico da sessão (espaço para reflexão, simbolização e elaboração das vivências do grupo).

A técnica utilizada como dispositivo psicoterapêutico, em seus diversos momentos, direcionou o grupo no sentido da organização psíquica. Gerou 
um ambiente que oportunizou a relação com o outro e, ao mesmo tempo, constituiu-se num espaço elaborativo. $O$ grupo se transformou num espaço para se legitimar afetos, como a agressividade, que encontrou dentro da prática de AF coletiva com bola uma possibilidade de expressão culturalmente aceita. Propiciou um cenário para o estabelecimento de processos de identificação, ao funcionar como elemento facilitador do contato intersubjetivo no aqui e agora. Dessa maneira, esse setting não convencional agiu no sentido de atenuar os sintomas do adoecimento mental severo. Posteriormente, investigou-se a técnica grupal, Grupo de Atividades Físicas, como dispositivo psicoterapêutico para pacientes psiquiátricos com adoecimento mental severo inseridos num Centro de Atenção Psicossocial (CAPS); (outpatients: pacientes em regime de atendimento ambulatorial) que fazia parte do mesmo serviço público de saúde mental. O grupo era aberto, heterogêneo quanto ao diagnóstico, gênero e idade; 16 pacientes, faixa etária de 27 a 56 anos. Concluiu-se que essa técnica grupal apresentava uma característica especial no que se refere à comunicação não verbal desenvolvida no momento da atividade física coletiva; esse contexto propiciava um "jogo" de possibilidades, em que o reconhecimento pelo "passar da bola" sinalizava para um contato intersubjetivo. $\mathrm{O}$ envolvimento na atividade grupal proposta remeteu a um movimento da libido em direção à realidade externa, noticiando um afastamento do processo de adoecimento mental severo (Zago, Terzis, \& Padilha, 2014). Um estudo também recente (Priebe, Savill, Ulrich, \& Wykes, 2013) avaliou a eficácia de uma forma de grupoterapia psicoterapia corporal, em inglês, body psychoterapy (BPT) em aliviar os sintomas negativos da esquizofrenia. A conclusão aponta que essa proposta interventiva pode ser útil e atrativa a esses pacientes devido à sua abordagem não usual e também ao serviço, pois demanda baixo investimento em termos de custo. Sinalizou que fatores não específicos podem ser responsáveis por pelo menos parte dos efeitos benéficos advindos da prática de AF, como desenvolver atividades em grupo. Outro estudo (Gomesa et al., 2014) verificou os efeitos da prática de atividade física em grupo (duas sessões por semana, durante 16 semanas) em termos de promoção de qualidade de vida para indivíduos com esquizofrenia (outpatients). Concluiu-se que esse programa de atividade física em grupo pode ser implantado exitosamente para pacientes com esquizofrenia não internados, atuando no sentido de incrementar sua qualidade de vida.

Sem dúvida, o grupo se constitui em dispositivo terapêutico efetivo para pacientes com SMI, como ilustra a história do surgimento da psicoterapia de grupo desde o século passado. No entanto a utilização de atividade física como elemento mediador da comunicação entre os elementos do grupo constituise numa nova proposta interventiva para o atendimento desses pacientes, 
especialmente em situações em que os recursos verbais estão comprometidos às vezes pelo mutismo ou por processos delirantes.

\subsection{Aderência dos pacientes com doença mental severa a programas de tratamento que incluem atividade física}

A literatura sugere que pacientes com SMI apresentam baixos níveis de participação em AF quando comparados a grupos controle de sujeitos que não se enquadram nessa categoria (Nyboe \& Lund, 2012). Nesse sentido, outro ponto discutido refere-se à importância de realizar-se a atividade com pacientes com SMI de maneira supervisionada. Embora os pacientes psiquiátricos demonstrem interesse em exercícios físicos (Giuliani et al., 2005; Richardison et al., 2005; Ussher et al., 2007; Zago \& Terzis, 2012), a aderência ao programa proposto está relacionada a um suporte técnico efetivo (Ussher et al., 2007; Zago \& Terzis, 2012). Os pacientes relatam que se exercitariam mais se a prática de AF fosse aconselhada por seu médico, o que corrobora a importância da participação dos cuidadores (médicos, psicólogos, enfermeiros, etc.) na adesão e assiduidade dos pacientes a essa proposta terapêutica. Por outro lado, apesar de os efeitos terapêuticos de AF virem sendo reconhecidos por profissionais da área da saúde mental, poucos recomendam essa prática a seus pacientes, pois haveria a crença de que essas discussões caberiam a especialistas em recreação física e fisiologistas (McEntee \& Halgin, 1996); aqueles com orientação psicanalítica têm sido relutantes em utilizar o exercício para fins terapêuticos, ou como adjunto às formas tradicionais de psicoterapia (O’Kelly et al., 1998). Porém um estudo que contou com a participação de 620 psicólogos sugere que muitos se consideram dispostos e potencialmente aptos em promover AF como parte do tratamento psicológico e desejariam participar em desenvolvimento profissional nessa área (Burton, Pakenham \& Brown, 2010). Além do que, a prática de AF em um procedimento estruturado para pacientes psiquiátricos apresenta-se como uma alternativa viável em unidades que atendem pacientes internados (Zago \& Terzis, 2012) ou em centros de atenção diária, como os centros de atenção psicossocial (Adamoli \& Azevedo, 2009; Zago et al., 2014).

A área de enfermagem está cada vez mais ciente do importante papel que exercem no encorajamento e assistência dos pacientes em relação à prática de AF em saúde mental, embora não tenham, por vezes, a preparação para desenvolver esse papel eficientemente (Happell, Phung-Platania, \& Scott, 2011). Analisando-se o nível de participação em atividade física de pacientes com SMI, há a compreensão de que o suporte social adequado pode atuar no sentido contrário às barreiras relativas à ansiedade e sintomas depressivos contribuindo, 
dessa maneira, para o aumento da participação (Bonsaksen, Fung, \& Tsang, 2011). Zago \& Terzis (2012) relataram a importância do papel dos cuidadores, ${ }^{4}$ principalmente, psicoterapeuta, técnico em enfermagem e educador físico, no sentido de secretariar os pacientes em direção ao estabelecimento de vínculo entre si e com a atividade grupal proposta que continha a prática coletiva de AF. Em estudo recente, discutiu-se a importância da integração da Educação Física como ciência auxiliar à Psiquiatria, segundo perspectiva interdisciplinar de tratamento em saúde mental (Melo, Oliveira, \& Vasconcelos-Raposo, 2014). Faz-se fundamental a promoção de diálogo e maior aproximação entre as diferentes áreas do conhecimento que dão suporte à atuação em saúde mental, a fim de que novas propostas interventivas sejam estruturadas e desenvolvidas numa perspectiva multidisciplinar.

\subsection{Percepção dos pacientes com doença mental severa sobre a prática de atividade física no programa de tratamento}

Em um estudo qualitativo, foram investigadas as experiências de usuários de serviços de saúde mental que tinham como parte do tratamento a participação em um programa de exercícios (sports therapy) (Crone \& Helen, 2008). Os resultados corroboram a consideração de que a terapia esportiva se constitui em um aceitável e benéfico adjunto ao tratamento convencional. Apontam a necessidade de uma maior compreensão teórica a esse respeito. Um estudo analisou as barreiras para a captação e aderência de pacientes com diagnóstico de esquizofrenia a programas de $\mathrm{AF}$ locados na comunidade. Os resultados dessa pesquisa qualitativa obtidos em entrevistas semiestruturadas com 27 pacientes colocam quatro barreiras: experiência restrita de engajamento em AF, impacto da doença e da medicação, efeitos da ansiedade e a influência das redes de suporte (Johnstone, Nicol, Donaghy, \& Lawrie, 2009). Outro estudo realizado por meio de grupos focais e entrevistas semiestruturadas com 34 pacientes ambulatoriais aponta as seguintes barreiras em participar de programas de AF: sintomas do adoecimento mental, medicação, ganho de peso, medo de condiçõos inseguras, medo de discriminação e interpretaçôes sobre o cumprimento do programa. Contudo os pacientes tinham a prática de $\mathrm{AF}$ como algo positivo e associado à saúde mental (McDevvitt, Snyder, Miller, \& Wilbur, 2006).

Outro estudo procurou estabelecer um entendimento a respeito do papel do esporte (futebol) e do exercício na recuperação de homens com adoecimento mental severo; mediante uma abordagem interpretativa de entrevistas semiestruturadas e diários de pesquisa (18 meses), apresentou o estudo de dois

4 Na extensão de toda a obra, usa-se o termo cuidador para designar profissionais da área de saúde (médicos, enfermeiros, psicólogos, assistentes sociais, etc.) (Osorio, 2007). 
casos (dois homens usuários de um serviço de reabilitação para pessoas com adoecimento mental severo): ( $\left.1^{\circ}\right)$ tinha experiência pessoal em participação esportiva e exercícios; (2o) estava disposto a participar da pesquisa. Ambos relataram benefícios psicológicos positivos em termos de humor e concentração, e sociais pertinentes à troca de experiências com outros e ao favorecimento de um espaço para acolher produções verbais. O esporte e o exercício eram centrais na identidade do primeiro participante e fundamentais para o seu senso de self. Para ele, regressar ao esporte e exercício após a remissão dos sintomas psicóticos representou o retorno a atividades imbuídas de sentido. No segundo caso, o esporte e o exercício foram usados como ferramentas ou veículos para resultados desejados que facilitaram atividades pessoais com sentido representando um começo e uso valoroso do tempo (Carless \& Douglas, 2008). Um estudo recente (Battaglia, et al., 2013) investigou os efeitos da prática de futebol na percepção da qualidade de vida e desempenho esportivo em sujeitos com psicose. A intervenção consistia em duas sessões de treino por semana (12 semanas). Os resultados apontam que a prática de futebol melhora a saúde psíquica e física de indivíduos com diagnóstico de esquizofrenia. Ao mesmo tempo, esse estudo demonstrou que a prática de AF desenvolvida por esse programa de treinamento de futebol reduziu a necessidade de medicação antipsicótica e, consequentemente, os efeitos adversos dessa prescrição para esses sujeitos. Um estudo que investigou a promoção do bem-estar em serviços de saúde mental, do ponto de vista dos usuários e dos profissionais, sugere que sejam feitas parcerias com locais da comunidade, como as que oportunizam a prática de AF (Owens, Crone, Kilgour, \& Ansari, 2010).

A prática de AF de maneira coletiva em um centro de convivência, isto é, fora do ambiente formal de tratamento, traz a possibilidade de aproximação dos participantes com as pessoas da comunidade. Ao mesmo tempo, a introdução de propostas interventivas deve estar alinhada à orientação de se inserir açóes terapêuticas na base territorial que compõe a rede de saúde da realidade social em questão.

\subsection{Integração da prática de $\mathrm{AF}$ em programas de tratamento de pacientes com SMI}

Richardison et al. (2005) realizaram uma revisão importante sobre a integração de AF nos serviços de saúde mental. Dentro dessa revisão, assinala-se que o exercício pode aliviar sintomas secundários de esquizofrenia, como depressão, baixa autoestima e retraimento social. Além disso, AF pode reduzir o isolamento social para as pessoas com SMI e ser uma via de promoção do engajamento 
dos indivíduos em serviços de saúde mental, oferecendo oportunidades seguras para interação social (Carter-Morris \& Faulkner, 2003; Zago \& Terzis, 2012). Aponta-se que a prática de AF representa um adjunto aceitável e benéfico ao tratamento usual para indivíduos com SMI (Crone \& Helen, 2008). Em revisão sistemática da literatura (Holley, Crone, \& Tyson, 2011), sugeriu-se que AF tem um efeito benéfico em alguns atributos associados ao bem-estar psicológico em indivíduos com esquizofrenia. Um dos estudos citados (Racinet \& Chevrollier, 1997) baseava-se num programa relativo à prática de futebol em hospital psiquiátrico. Essa revisão também apontou a necessidade de maiores pesquisas em relação a esse tema. Em revisão crítica recente que apresenta trabalhos relativos ao impacto do exercício na saúde mental e qualidade de vida de pessoas com SMI publicados no período entre 1998 a 2009 (Alexandratos, Barnett, \& Thomas, 2012), os autores concluem que o exercício pode contribuir na melhora em termos de sintomatologia: humor, concentração, padrões de sono e sintomas psicóticos. Ao mesmo tempo, também a prática de exercícios pode melhorar a qualidade de vida por meio da interação social, uso do tempo preenchido por sentido, atividade que apresenta propósito e empoderamento. Os autores recomendam pesquisas que possam trazer mais subsídios em relação à prática de exercícios pertinentes às necessidades específicas dessa população. Enfatizam ainda que os estudos devem direcionar seu foco para avaliações psicológicas que pudessem prover maior evidência sobre o uso dessa terapia. Nesse sentido, estudos (Zago \& Terzis, 2012; Zago et al., 2014) apontam que a psicoterapia de grupo de base analítica pode contribuir trazendo uma compreensão sobre a maneira como a subjetividade de pacientes com SMI se coloca dentro do "jogorelacional" mediada por atividade física em grupo e, mais profundamente, uma leitura da realidade psíquica inconsciente compartilhada que se desenvolve por meio da comunicação interdiscursiva.

\section{CONCLUSÕES}

Conclui-se que a prática de AF pode contribuir para melhora de humor, estado de alerta, concentração, padrões de sono e sintomas psicóticos; pode aliviar sintomas secundários, como baixa autoestima e retraimento social. Há evidência também de benefícios psicológicos da prática de $\mathrm{AF}$ em pacientes depressivos. Os pacientes veem AF positivamente e gostariam de serem ativos e melhorar a saúde mental. Barreiras apontadas pelos pacientes foram: sintomas do adoecimento mental, efeitos da medicação, fadiga, ganho de peso por medicação, medo de discriminação e questôes relativas à segurança e falta de suporte profissional. $\mathrm{O}$ contexto grupal aparece como um ambiente favorecedor da prática de AF. Os profissionais de saúde mental têm sido uma fonte subutilizada na promoção de 
AF. A literatura considera AF como uma intervenção que pode ser útil para a promoção da saúde mental e bem-estar, portanto uma estratégia em reabilitação psicossocial para pessoas com SMI. Faz-se importante, dentro da noção de território, estabelecer parcerias com centros comunitários, de convivência, a fim de ofertar aos pacientes mais espaços possíveis para a prática de AF.

Há a necessidade da elaboração de mais estudos sobre as repercussões psíquicas da AF como intervenção terapêutica para pacientes com adoecimento mental severo. Entende-se que programas de tratamento que incluem AF podem ser direta ou indiretamente terapêuticos para esses pacientes, conforme seu objetivo e delineamento. Além disso, a prática de AF pode ser um elemento mediador de relaçôes intersubjetivas em dispositivos estruturados em situação de grupo com finalidade psicoterapêutica. Nesse sentido, a psicoterapia de grupo, e mais especificamente a de base analítica, pode contribuir na compreensão dos fenômenos psíquicos emergentes no desenvolvimento de AF no contexto coletivo.

Destaca-se a importância do papel dos profissionais na utilização da prática de AF como ferramenta viável, de baixo custo e potencialmente terapêutica no tratamento de pacientes com doença mental severa, quer em centros de atenção diária ou em situação de cuidado intensivo. A presença da prática de AF em saúde mental depende da maneira como os gerentes entendem as práticas de atendimento e a elaboração dos programas de tratamento, da atribuição de valor que os profissionais cuidadores dão a essa prática, como também da disponibilidade de todos em dar suporte aos pacientes no processo de adesão a essa proposta interventiva. 


\section{REFERÊNCIAS}

Adamoli, A. N. \& Azevedo, M. R. (2009, fevereiro). Padrões de atividade física de pessoas com transtornos mentais e de comportamento. Ciência \& Saúde Coletiva, 14(1), 243-251.

Alexandratos, K., Barnett, F. \& Thomas, Y. (2012). The impact of exercise on the mental health and quality of life of people with severe mental illness: a critical review. British Journal of Occupation Therapy, 75(2), 48-73.

Battaglia, G., Alesi, M., Inguglia, M., Roccella, M., Caramazza, G. \& Bellafiore, M. \& Palma, A. (2013). Soccer practice as an add-on treatment in the management of individuals with a diagnosis of schizophrenia. Neuropsychiatric Disease And Treatment, 9, 595-603.

Bonsaksen, T., Fung, K. \& Tsang, H. (2011). Participation in physical activity among inpatients with severe mental illness. International Journal of Therapy \& Rehabilitation, 18(2), 91-9.

Broocks, A. (2005). Physical training in the treatment of psychological disorders. Bundesgesundheitsblatt Gesundheitsforschung Gesundheitsschutz, 48(8), 914921.

Broocks, A. \& Sommer, M. (2005, novembro). Psychische sportwirkungen. I psychological effects of sport. Deutsche Zeitschrift fuer Sportmedizin, 56(11), 393-94.

Burton, N. W., Pakenham, K. I. \& Brown, W. J. (2010). Are psychologists willing and able to promote physical activity as part of psychological treatment? International Journal Behavior Medicine, 17, 287-297.

Carless, D. \& Douglas, K. (2008). The role of sport and exercise in recovery from serious mental illness: two case studies. International Journal of Men's Health, 7(2), 137-156.

Carter-Morris, P. \& Faulkner, G. (2003). A football project for service users: the role of football in reducing social exclusion. Journal of Mental Health Promotion, 2, 24-30.

Crone, D. \& Helen, G. (2008). "I khow it is only exercise, but t me it is something that keeps me going": a qualitative approach to understanding mental health service users' experiences of sports therapy. International Journal of Mental Health Nursing, 17, 197-207. 
Faulkner, G. \& Biddle, S. (1999). Exercise as an adjunct treatment for schizophrenia: a review of the literature. Journal of Mental Health, 8, 441-457.

Faulkner, G. \& Carless, D. (2006). Physical activity in the process of psychiatric rehabilitation: theoretical and methodological issues. Psychiatric Rehabilitation Journal, 29(4), 258-66.

Faulkner, G., Cohn, T. \& Remington, G. (2006, fevereiro). Validation of a physical activity assessment tool for individuals with schizophrenia. Schizophrenia Research, 82(2-3), 225-231.

Faulkner, G. \& Sparkes, A. (1999). Exercise as therapy for schizophrenia: an ethnographic study. Journal of Sport and Exercise Psychology, 21(1), 52-69.

Fogarty, M. \& Happell, B. (2005). Exploring the benefits of an exercise program for people with schizophrenia: a qualitative study. Issues in Mental Health Nursing, 26(3), 341-351.

Giuliani, A., Micacchi, G. \& Valenti, M. (2005). L'attività motoria nei servizi di salute mentale: evidenze scientifiche e linne guida. Italian Journal of Sport Sciences, 12(2),116-124.

Gomesa, E., Bastos, T., Probst, M., Ribeiro, J. C., Silva, G. \& Corredeira, R. (2014). Effects of a group physical activity program on physical fitness and quality of life in individuals with schizophrenia. Mental Health and Physical Activity, 7(3), 155-162.

Happell, B., Phung-Platania, C. \& Scott, D. (2011). Placing physical activity in mental health care: a leadership role for mental health nurses. International Journal of Mental Health Nursing, 20(5), 310-318.

Holley, J., Crone, D. \& Tyson, P. (2011). The effects of physical activity on psychological well-being for those with schizophrenia: a systematic review. British Journal of Clinical Psychology, 50(1), 80-105.

Hutchinson, D. S., Skrinar, G. S. \& Cross, C. (1999). The role of improved physical fitness in rehabilitation and recovery. Psychiatric Rehabilitation Journal, $22,355-59$.

Insel, T. (2013, 31 julho). Getting serious about mental illnesses. National Institute of Mental Health, 58(96), 29422-29425. Recuperado a partir de http://www. nimh.nih.gov/about/director/2013/getting-serious-about-mental-illnesses. shtml 
Johnstone, R., Nicol, K., Donaghy, M. \& Lawrie, S. (2009). Barriers to uptake of physical activity in community-based patients with schizophrenia. Journal of Mental Health, 18(6), 523-532.

Leibovich, P. \& Iancu, I. (2007, dezembro). Physical exercise among psychiatric patients: physical and mental effects. Harefuah, 146(12), 975-979, 996.

McDevitt, J., Robinson, N. \& Forest, D. (2005, março). A group-based walking program at a psychiatric rehabilitation center. Psychiatric Services, 56(3), 35455.

McDevitt, J., Snyder, M., Miller, A. \& Wilbur, J. (2006). Perceptions of barriers and benefits to physical activity among outpatients in psychiatric rehabilitation. Journal of Nursing Scholarship, 38(1), 50-55.

McEntee, D. J. \& Halgin, R. P. (1996). Therapists' attitudes about addressing the role of exercise in psychotherapy. Journal of Clinical Psychology, 52(1), 4860 .

Melo, L. G. dos S. C., Oliveira, K. R. da S. G. de \& Vasconcelos-Raposo, J. (2014). A educação física no âmbito do tratamento em saúde mental: um esforço coletivo e integrado. Revista Latino-americana de Psicopatologia Fundamental, 17(3), 501-514.

Meyer, T. \& Broocks, A. (2000, outubro). Therapeutic impact of exercise on psychiatric diseases: guidelines for exercise testing and prescription. Sports Medicine, 30(4), 269-279.

Nyboe, L. \& Lund, H. (2012, maio). Low levels of physical activity in patients with severe mental illness. Nordic Journal of Psychiatry, 67(1), 43-46.

O’Kelly, J. G., Piper, W. E., Kerber, R. \& Fowler, J. (1998). Exercise groups in an insight-oriented, evening treatment program. International Journal of Group Psychotherapy, 48(1), 85-98.

Osório, L. C. (2007). Grupoterapias, abordagens atuais. Porto Alegre: Artmed.

Owens, C., Crone, D., Kilgour, L. \& El Ansari, W. (2010). The place and promotion of well-being in mental health services: a qualitative investigation. Journal of Psychiatric and Mental Health Nursing, 17(1), 1-8.

Priebe, S., Savil, M., Ulrich, R. \& Wykes, T. (2013, janeiro). Effectiveness and cost-effectiveness of body psychotherapy in the treatment of negative symptoms 
of schizophrenia - a multi-centre randomized controlled trial. BioMedCentral Psychiatry, 13(26), 1-8.

Racient, G. \& Chevrollier, J. P. (1997). Football in a psychiatric hospital: Results in five schizophrenics. Societe Medico-Psychologique, 155(8), 530-33.

Holley, J., Crone, D. \& Tyson P. (2011). The effects of physical activity on psychological well-being for those with schizophrenia: a systematic review. Britisch Journal of Clinical Psychology, 50(1), 80-105.

Richardison, C. R., Faulkner, G., McDevitt, J., Skrinar G. S., Hutchinson, Sc. D. \& Piette, J. D. (2005, março). Integrating physical activity into mental health services for persons with serious mental illness. Psichiatric Services, 56(3), 324-331.

Scott, D. \& Happell, B. (2011). The high prevalence of poor physical health and unhealthy lifestyle behaviours in individuals with severe mental illness. Issues in Mental Health Nursing, 32(9), 589-597.

Skrinar, G. S., Unger, K. V., Hutchinson, D. S. \& Faigenbaum, A. D. (1992). Effects of exercise training in young adults with psychiatric disabilities. Canadian Journal of Rehabilitation, 5(3), 151-157.

Smith, S., Yeomans, D., Busche, C. J. P., Eriksson, C., Harrison, T., Holmes, R., . . Sullivan, G. (2007). A well-being programme in severe mental illness. Baselie findings in a UK cohort. Clinical Practice, 61(12),1971-1978.

Soundy, A., Faulkner, G. \& Taylor, A. (2007). Exploring variability and perceptions of lifestyle physical activity among individuals with severe and enduring mental health problems: a qualitative study. Journal of Mental Health, 16(4), 493-503.

Trivedi, M. H., Greer, T. L., Grannemann, B. D., Chambliss, H. O. \& Jordan, A. N. (2006, julho). Exercise as an augmentation strategy for treatment of major depression. Journal of Psychiatric Practice, 12(4), 205-213.

Ussher, M, Stanbury, L., Cheeseman, V. \& Faulkner, G. (2007). Physical activity preferences and perceived barriers to activity among persons with severe mental illness in the United Kingdom. Psychiatric Services, 58(3), 405-408.

Vancamfort, D., Probst, M., Skjaerven, L. H., Matamoros, D. C., Gyllensten, A. L., Conesa, A. G., . . . Hert, M. de. (2012). Systematic review of the benefits of physical therapy within a multidisciplinary care approach for people with 
schizophrenia. Research Report, 92(1), 11-23.

World Health Organization. (2010). Global recommendations on physical activity for health. Geneva: World Health Organization. Recuperado a partir de http:// whqlibdoc.who.int/publications/2010/9789241599979_eng.pdf

Zago, M. C. \& Terzis, A. (2012). O jogo-relacional de um grupo de pacientes psicóticos em atividade física: um estudo psicanalítico. Psicologia, 16(2), 6785 .

Zago, M. C., Terzis, A. \& Padilha, B. M. (2014). Comunicação inconsciente entre singular e plural: grupo de pacientes psiquiátricos. Revista da Sociedade de Psicoterapias Analíticas Grupais do Estado de São Paulo, 15(1), 4-16. 